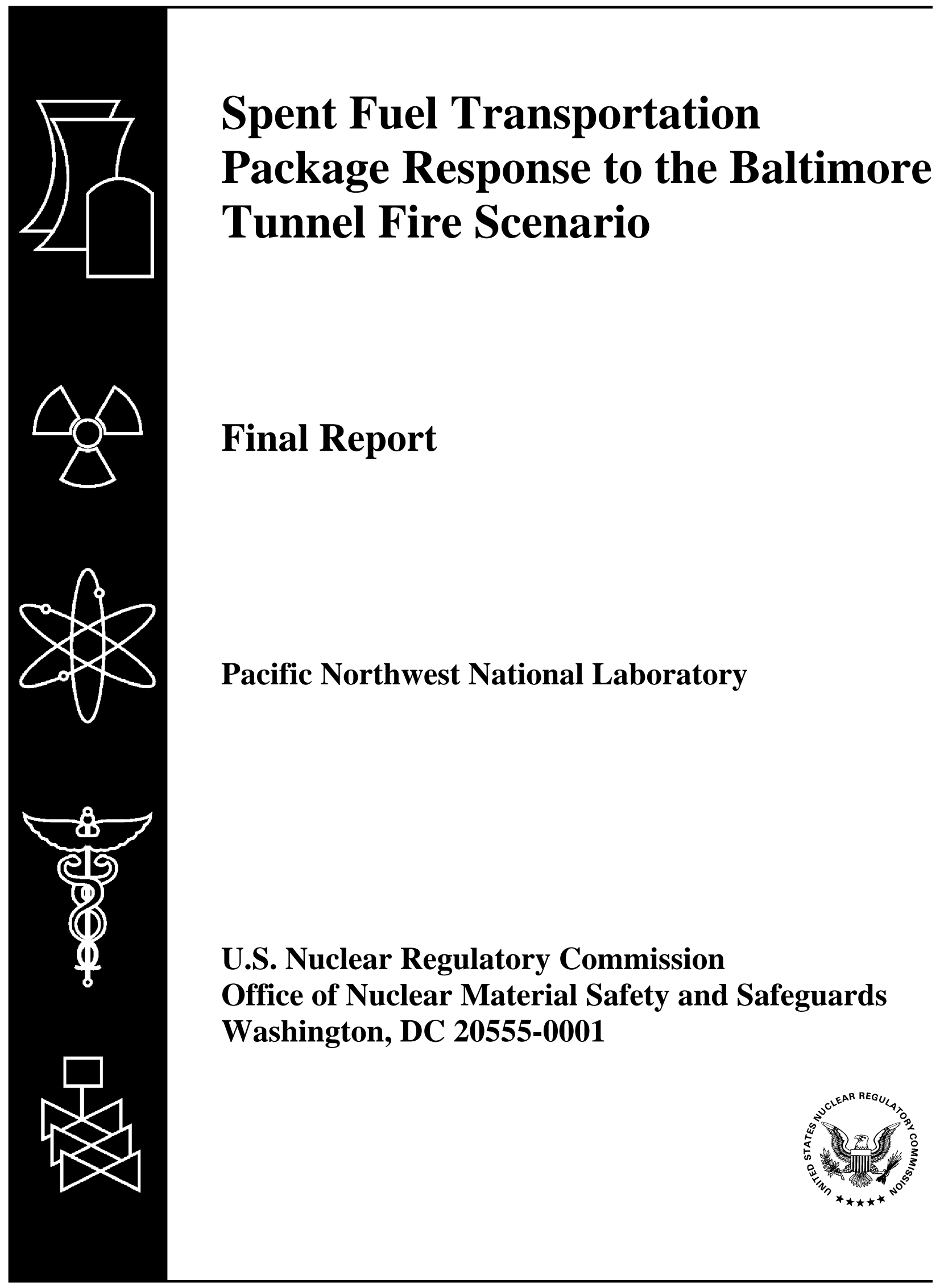


NUREG/CR-6886, Rev. 1

PNNL-15313

\section{Spent Fuel Transportation Package Response to the Baltimore Tunnel Fire Scenario}

\section{Final Report}

Manuscript Completed: October 2006

Date Published: November 2006

Prepared by

H.E. Adkins, Jr., J.M. Cuta, B.J. Koeppel, A.D. Guzman (PNNL)

C.S. Bajwa (NRC)

Pacific Northwest National Laboratory

902 Battelle Boulevard

Richland, WA 99352

\section{A. Hansen, NRC Project Manager}

\section{Prepared for}

Division of Spent Fuel Storage and Transportation

Office of Nuclear Material Safety and Safeguards

U.S. Nuclear Regulatory Commission

Washington, DC 20555-0001

Job Code J5167

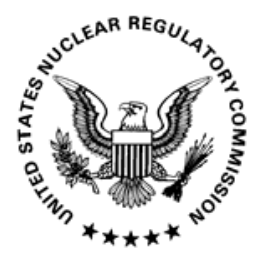




\section{ABSTRACT}

On July 18, 2001, a freight train carrying hazardous (non-nuclear) materials derailed and caught fire while passing through the Howard Street railroad tunnel in downtown Baltimore, Maryland. The United States Nuclear Regulatory Commission (USNRC), one of the agencies responsible for ensuring the safe transportation of radioactive materials in the United States, undertook an investigation of the train derailment and fire to determine the possible regulatory implications of this particular event for the transportation of spent nuclear fuel by railroad.

The USNRC met with the National Transportation Safety Board (NTSB) to discuss the details of the accident and the ensuing fire. Following these discussions, the USNRC assembled a team of experts from the National Institute of Standards and Technology (NIST), the Center for Nuclear Waste Regulatory Analyses (CNWRA), and Pacific Northwest National Laboratory (PNNL) to determine the thermal conditions that existed in the Howard Street tunnel fire and analyze the potential effects of those conditions on various spent nuclear fuel transportation package designs.

The Fire Dynamics Simulator (FDS) code developed by NIST was used to determine the thermal environment in the Howard Street tunnel during the fire. The FDS results were used as boundary conditions for the COBRA-SFS and ANSYS ${ }^{\circledR}$ computer models developed to evaluate the thermal performance of different package designs. The staff concluded that larger transportation packages resembling the TransNuclear Model No. TN-68 and HOLTEC Model No. HI-STAR 100 would withstand a fire with thermal conditions similar to those that existed in the Baltimore tunnel fire event with only minor damage to peripheral components. This is due to their sizable thermal inertia and design specifications in compliance with currently imposed regulatory requirements.

For the TN-68 and the NAC International Model No. LWT (legal weight truck) transportation package, the maximum temperatures predicted in the regions of the lid and the vent and drain ports exceed the seals' rated service temperatures, making it possible for a small release to occur, due to CRUD that might spall off the surfaces of the fuel rods. While a release is not expected to occur for these conditions, any release that could occur would be very small due to a number of factors. These include (1) the tight clearances maintained between the lid and cask body by the closure bolts, (2) the low pressure differential between the package interior and exterior, (3) the tendency of such small clearances to plug, and (4) the tendency of CRUD particles to settle or plate out.

USNRC staff evaluated the radiological con-sequences of the package responses to the Baltimore tunnel fire. The analysis indicates that the regulatory dose rate limits specified in 10 CFR 71.51 for accident conditions would not be exceeded by releases or direct radiation from any of these packages in this fire scenario. All three packages are designed to maintain regulatory dose rate limits even with a complete loss of neutron shielding (as documented in their respective SAR analyses.) While highly unlikely, the NAC LWT could experience some decrease in gamma shielding due to slump in the lead as a consequence of this fire scenario, but a conservative analysis shows that the regulatory dose rate limits would not be exceeded. 
The results of this evaluation also strongly indicate that neither spent nuclear fuel (SNF) particles nor fission products would be released from a spent fuel transportation package carrying intact spent fuel involved in a severe tunnel fire such as the Baltimore tunnel fire. None of the three package designs analyzed for the Baltimore tunnel fire scenario (TN-68, HI-STAR 100, and NAC LWT) experienced internal temperatures that would result in rupture of the fuel cladding. Therefore, radioactive material (i.e., SNF particles or fission products) would be retained within the fuel rods.

There would be no release from the HI-STAR 100, because the inner welded canister remains leak tight. While a release is unlikely, the potential releases calculated for the TN-68 rail package and the NAC LWT truck package indicate that any release of CRUD from either package would be very small - less than an $A_{2}$ quantity (see Section 8.2.) 


\section{CONTENTS}

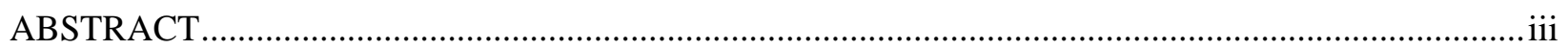

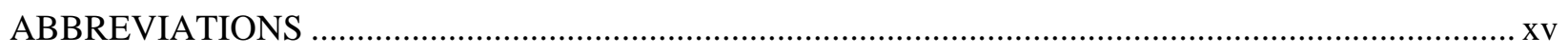

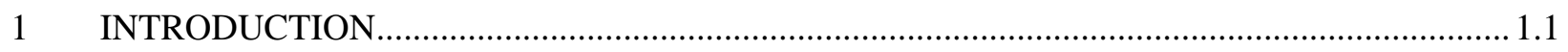

1.1 Evaluation of Tunnel Fire Characteristics .................................................................... 1.1

1.2 Review of Rail Transportation Accidents .................................................................. 1.3

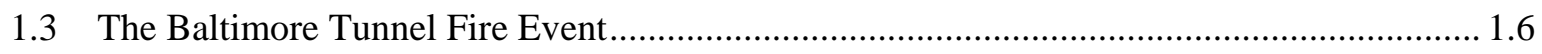

1.4 Implications of the Baltimore Tunel Fire for Transportation of Spent Nuclear Fuel ............ 1.8

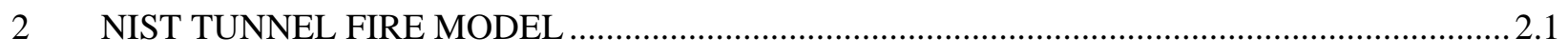

$3 \quad$ CNWRA MATERIALS FIRE EXPOSURE ANALYSIS .......................................................... 3.1

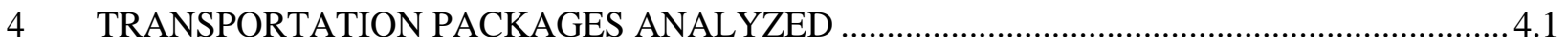

4.1 TransNuclear TN-68 SNF Transportation Package ........................................................ 4.1

4.2 HOLTEC HI-STAR 100 SNF Transportation Package ................................................... 4.3

4.3 NAC LWT SNF Transportation Package ....................................................................... 4.4

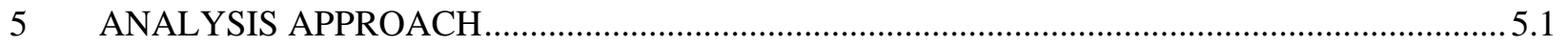

5.1 Modeling SNF Packages within Tunnel................................................................... 5.1

5.2 Model of TN-68 Transportation Package ....................................................................... 5.5

5.3 Model of HI-STAR 100 Transportation Package .............................................................. 5.8

5.4 Model of NAC LWT Transportation Package ............................................................ 5.17

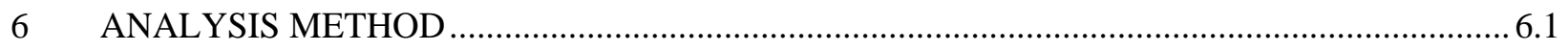

6.1 Modeling Assumptions and Boundary Conditions.......................................................... 6.1

6.1.1 Boundary Temperatures from FDS ...................................................................... 6.3

6.1.2 Convection and Radiative Heat Transfer Boundary Conditions ................................ 6.5

6.1.3 Extrapolated Boundary Conditions for Long-Term Cool Down............................... 6.7

6.1.4 Heat Transfer through NAC LWT Liquid Neutron Shield ...................................... 6.10 
6.1.5 Heat Transfer through NAC LWT Lead Gamma Shield.

6.2 Initial System Component Temperatures ..................................................................... 6.13

6.3 Tunnel Fire Evaluations of Rail Packages...................................................................... 6.21

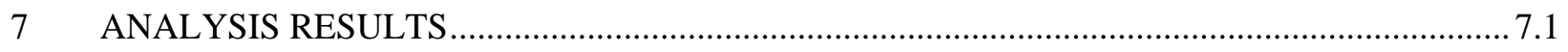

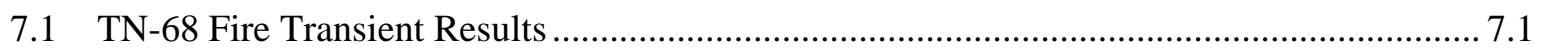

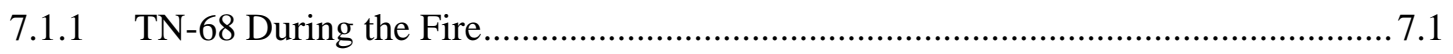

7.1.2 TN-68 Short-Term Post-Fire Response................................................................ 7.4

7.1.3 TN-68 Long-Term Post-Fire Response …............................................................. 7.9

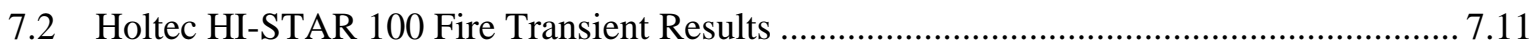

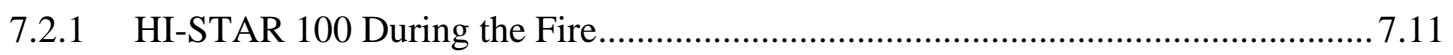

7.2.2 HI-STAR 100 Short-Term Post-Fire Response .........................................................13

7.2.3 HI-STAR 100 Long-Term Post-Fire Response........................................................ 7.17

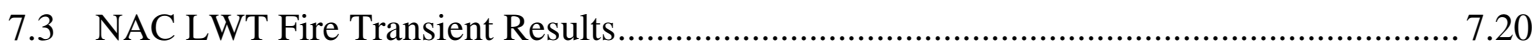

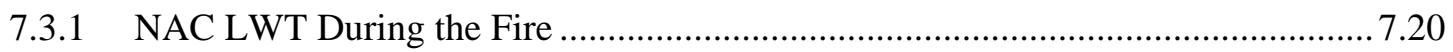

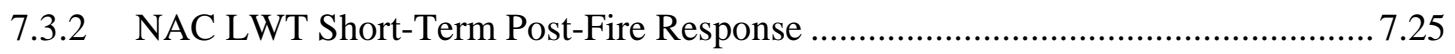

7.3.3 NAC LWT Long-Term Post-Fire Response.......................................................... 7.28

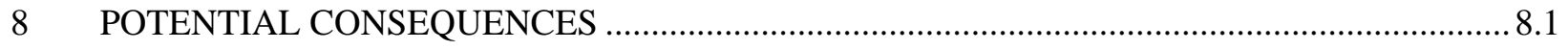

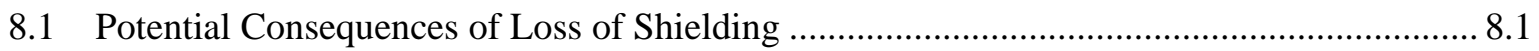

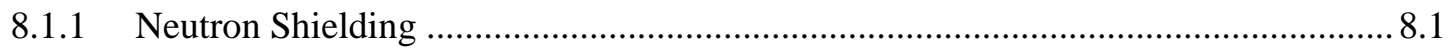

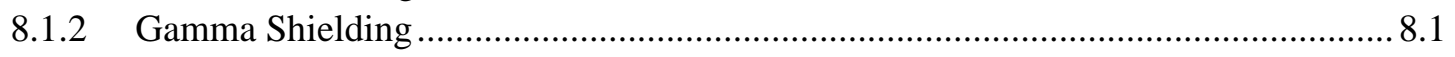

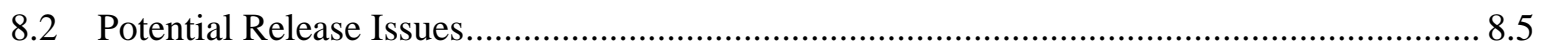

8.2.1 Seal Performance and Potential Leak Paths .............................................................. 8.6

8.2.2 Potential Release from the HI-STAR 100 Package..................................................... 8.6

8.2.3 Potential Release from the TN-68 Package........................................................... 8.7

8.2.4 Potential Release from the NAC LWT Package .................................................... 8.9

8.2.5 Potential Releases from Packages Carrying Failed Fuel ........................................ 8.11

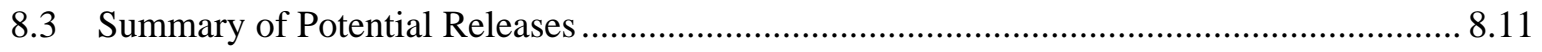

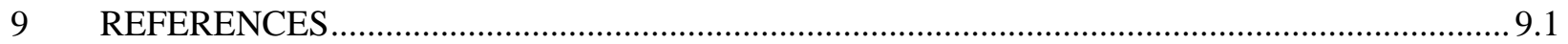




\section{FIGURES}

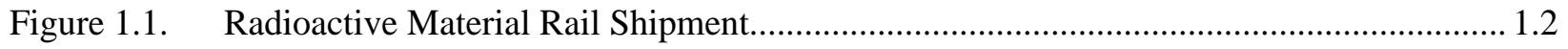

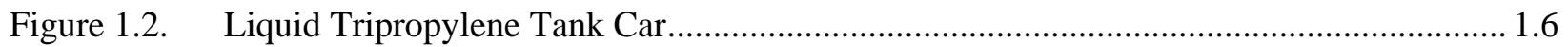

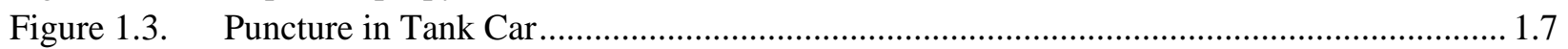

Figure 4.1. Cross-section of TN-68 Package (drawing 972-71-3 Rev. 4, "TN-68 Packaging General

Arrangement: Parts List and Details").......................................................................... 4.2

Figure 4.2. TransNuclear TN-68 Spent Fuel Transportation Package................................................... 4.2

Figure 4.3. HOLTEC HI-STAR 100 Spent Fuel Package ............................................................. 4.3

Figure 4.4. Spent Fuel Transportation Package on Rail car........................................................... 4.4

Figure 4.5. NAC LWT Transport Package (without ISO container) …............................................ 4.5

Figure 4.6. NAC LWT Transport Package (within ISO container) …............................................... 4.5

Figure 5.1. C Configuration of Derailed Train in Vicinity of Fire ..................................................... 5.2

Figure 5.2. $\quad$ Possible Orientation of Derailed Train in Tunnel ......................................................... 5.2

Figure 5.3. Dimensions of Howard Street Tunnel with Tank Car on Track ....................................... 5.3

Figure 5.4. Cross-section of Tunnel (diagram not to scale) Showing "Top", "Side”, and "Bottom”

Regions for Fire Boundary Temperatures ...................................................................... 5.5

Figure 5.5. C COBRA-SFS Model of TN-68 Basket and Support Rails............................................... 5.6

Figure 5.6. ANSYS HI-STAR 100 Package Analysis Model Element Plot..................................... 5.10

Figure 5.7. Cross-section of Package, Cradle, and Rail Car Section .............................................. 5.10

Figure 5.8. Cross-section of HI-STAR Package and MPC-24 Canister ......................................... 5.11

Figure 5.9. Close-up of Package Cross-section ........................................................................... 5.11

Figure 5.10. Close-up of Canister Basket and Fuel Compartment (without helium elements) ............ 5.12

Figure 5.11. Close-up of Canister Basket and Fuel Compartment (with helium elements) ................. 5.12

Figure 5.12. Complete Impact Limiter (Except Skin) .................................................................... 5.14

Figure 5.13. Impact Limiter Skin and Primary Support Structure .................................................... 5.14

Figure 5.14. Surfaces Defined for Interaction with “Top” Gas Region............................................ 5.15

Figure 5.15. Surfaces Defined for Interaction with "Side” Gas Region ............................................. 5.16

Figure 5.16. Surfaces Defined for Interaction with "Bottom” Gas Region ...................................... 5.16

Figure 5.17. ANSYS NAC LWT Package Analysis Model Element Plot........................................... 5.18

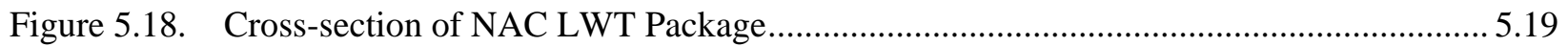

Figure 5.19. NAC LWT Package Geometry .............................................................................. 5.19

Figure 5.20. Z Zones for Convection Heat Transfer Within the ISO Container .................................... 5.22

Figure 5.21. Zones for External Radiation Between ISO Container and Tunnel Surfaces.................. 5.22

Figure 6.1. BTF Peak Transient Ambient Air Temperatures (smoothed values, NIST 20-m data) ..... 6.4

Figure 6.2. BTF Peak Transient Tunnel Surface Temperatures for Floor, Walls, and Ceiling (smoothed values, NIST 20-m data).................................................................................... 6.4

Figure 6.3. BTF Peak Transient Horizontal Velocities near Package Surface (smoothed values, NIST

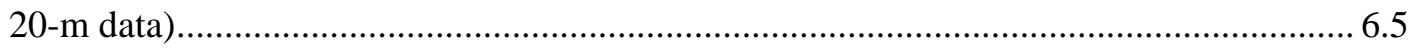

Figure 6.4. Convection Heat Transfer Coefficients at Package Surface from NIST 20 m Air Temperature and Velocity Predictions .....

Figure 6.5. BTF Peak Transient Air Temperatures for Top, Side, and Bottom Regions (NIST Data Set and Extrapolated Values) 6.8 
Figure 6.6. BTF Peak Transient Surface Temperatures for Floor, Walls, and Ceiling (NIST Data Set

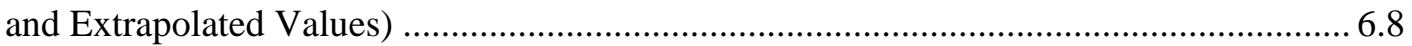

Figure 6.7. Nusselt Number for Heat Transfer in Liquid Neutron Shield ....................................... 6.11

Figure 6.8. Effective Conductivity of Neutron Shield Tank Contents............................................ 6.12

Figure 6.9. Effective Conductivity of Expansion Tank Contents .................................................. 6.12

Figure 6.10. Solid and Molten Lead: Enthalpy as a Function of Temperature .................................. 6.14

Figure 6.11. Solid and Molten Lead: Thermal Conductivity as a Function of Temperature ............... 6.14

Figure 6.12. HI-STAR 100 Package Hot-Normal Condition Temperature Distribution ....................... 6.16

Figure 6.13. LWT Package Hot-Normal Condition Temperature Distribution (2.5 kW Decay Heat) ... 6.19

Figure 6.14. LWT Package Normal Condition Temperature Distribution (2.5 kW Decay Heat) ........ 6.21

Figure 7.1. Maximum Temperature Histories for TN-68 Components During Fire Transient ............ 7.2

Figure 7.2. Temperature Profiles Top-To-Bottom Through TN-68 Package During Fire Transient ... 7.3

Figure 7.3. Maximum Temperature Histories for TN-68 Package Components During First $30 \mathrm{hr}$ of

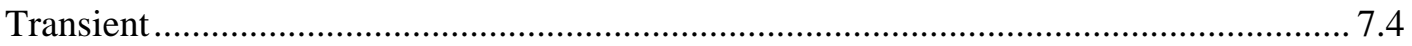

Figure 7.4. Maximum TN-68 Package Surface Temperatures Compared to NIST Boundary Condition

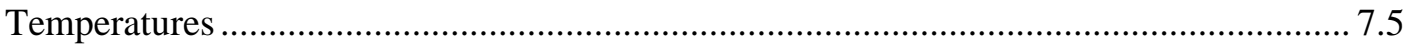

Figure 7.5. Peak Fuel Cladding Temperature History in TN-68 During First 50 hr of Transient ........ 7.6

Figure 7.6. Maximum Global TN-68 Closure/Port Seal Temperature History for 30-hr Transient ..... 7.8

Figure 7.7. Temperature Profiles Top-To-Bottom Through TN-68 Package Axis to 30 hrs............... 7.8

Figure 7.8. Maximum Temperature Histories for TN-68 Package Components During $300 \mathrm{hr}$ of

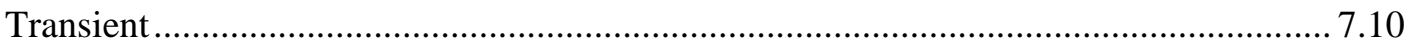

Figure 7.9. Maximum Temperature Histories for HI-STAR 100 Package Components During Fire

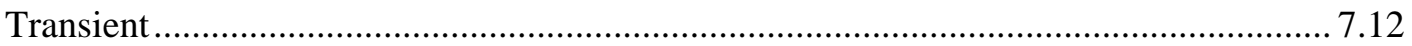

Figure 7.10. Maximum Temperature Histories for HI-STAR 100 Package Components During First 30

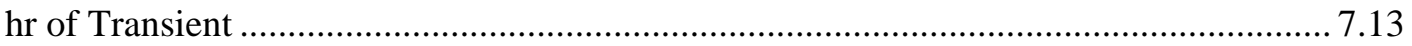

Figure 7.11. Maximum HI-STAR 100 Package Surface Temperature Compared to NIST Boundary

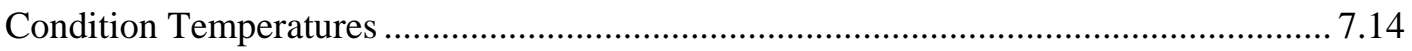

Figure 7.12. Peak Fuel Clad Temperature History in HI-STAR 100 During First 50 hr of Transient . 7.15

Figure 7.13. Maximum Global HI-STAR 100 Closure/Port Seal Temperature History During First $30 \mathrm{hr}$

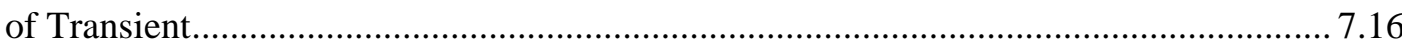

Figure 7.14. Maximum Temperature Histories for HI-STAR 100 Package Components During 300 hrs

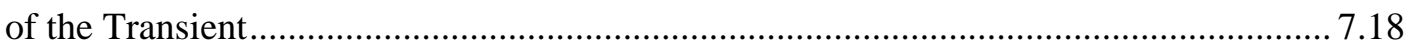

Figure 7.15. NAC LWT Component Maximum Temperature Histories During Fire Transient .......... 7.21

Figure 7.16. Lumped Fuel Assembly Temperature Distribution 7 hr into Transient .......................... 7.22

Figure 7.17. Temperature History of Selected Elements in Cask Body Lead in NAC LWT Package. 7.23

Figure 7.18. NAC LWT Package Component Maximum Temperature Histories for First 30 hours of

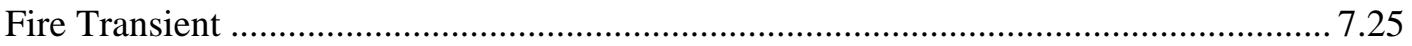

Figure 7.19. Maximum ISO Container Surface Temperature History Compared with NIST Boundary

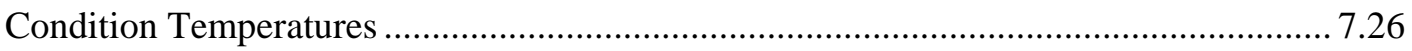

Figure 7.20. $\quad$ Peak and Average Fuel Cladding Temperature Histories for NAC LWT Package During First $30 \mathrm{hr}$ of Fire Transient........................................................................................ 7.27

Figure 7.21. Maximum Seal Temperature Histories for Drain/Vent Ports and Package Lid During First

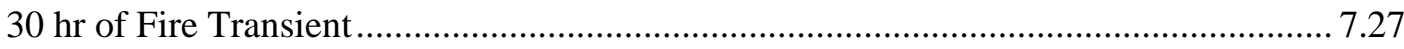


Figure 7.22. NAC LWT Package Component Maximum Temperature Histories During 300 hr

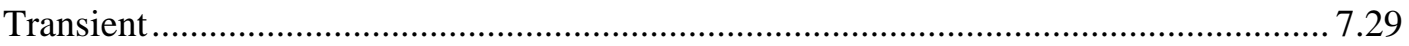

Figure 8.1. Lead Shielding Configuration in the NAC LWT Before and After Lead Melting ............ 8.4 


\section{TABLES}

Table 1.1. Comparison of Various Hydrocarbon Liquids............................................................ 1.8

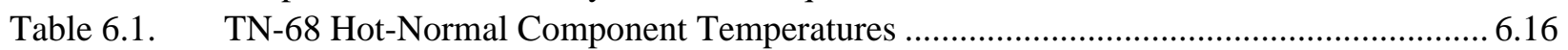

Table 6.2. HOLTEC HI-STAR 100 Hot-Normal Component Temperatures........................................... 6.17

Table 6.3. NAC LWT Component Temperatures at Various Decay Heat Loads.............................. 6.18

Table 6.4. NAC LWT Component Temperatures at $2.5 \mathrm{~kW}$ Decay Heat and $130^{\circ} \mathrm{F}$ Ambient......... 6.20

Table 6.5. NAC LWT Pre-Fire Component Temperatures at $2.5 \mathrm{~kW}$ Decay Heat Load and $100^{\circ} \mathrm{F}$

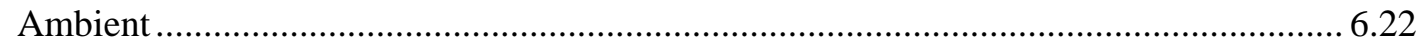

Table 7.1. TN-68 Peak Component Temperatures During Fire Transient ...................................... 7.11

Table 7.2. HOLTEC HI-STAR 100 Peak Component Temperatures During Fire Transient............ 7.19

Table 7.3. NAC LWT Lead Shielding Response to Fire Transient ................................................ 7.23

Table 7.4. NAC LWT Peak Component Temperatures During Fire Transient............................... 7.28

Table 8.1. $\quad$ Potential Dose Estimate from NAC LWT with Reduced Shielding ................................. 8.4

Table 8.2. $\quad$ Assumptions Used for Release Estimate for TN-68 Package ........................................... 8.8

Table 8.3. Potential Release Estimate for TN-68 Package ................................................................ 8.9

Table 8.4. Assumptions Used for Release Estimate for NAC LWT Package ................................. 8.10

Table 8.5. Potential Release Estimate for NAC LWT Package ........................................................ 8.10 


\section{APPENDICES}

APPENDIX A - Material Properties for COBRA-SFS Model of TN-68 Package ................................. A.1

APPENDIX B - Material Properties for ANSYS Model of HI-STAR 100 Package .................................1

APPENDIX C - Material Properties for ANSYS Model of Legal Weight Truck Package .......................1

APPENDIX D - Boundary Conditions from FDS Simulation of Fully Ventilated Fire Scenario ........... D.1

APPENDIX E - Blackbody Viewfactors for COBRA-SFS Model of TN-68 Package...............................1

APPENDIX F - HOLTEC HI-STAR 100 Component Temperature Distributions .................................F.1

APPENDIX G - Summary of Staff Responses to Public Comments Received from Federal Register

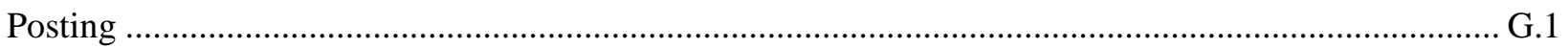




\title{
ABBREVIATIONS
}

\author{
APDL $\quad$ ANSYS ${ }^{\circledR}$ Parametric Design Language \\ BTF $\quad$ Baltimore Tunnel Fire \\ BWR Boiling Water Reactor \\ CFD Computational Fluid Dynamics \\ CoC Certificate of Compliance \\ CNWRA Center for Nuclear Waste Regulatory Analyses \\ CRUD Chalk River Unknown Deposit (generic term for various residues deposited on fuel rod \\ surfaces, originally coined by Atomic Energy of Canada, Ltd. (AECL) to describe deposits \\ observed on fuel removed from the test reactor at Chalk River.) \\ DOT Department of Transportation \\ FDS Fire Dynamics Simulator (computational fluid dynamics computer code) \\ FEA Finite Element Analysis \\ HLW High Level Waste \\ IAEA International Atomic Energy Agency \\ ISO International Organization for Standardization (The International Organization for \\ Standardization has decreed the use of the initials ISO for reference to the organization, \\ regardless of the word order of the organization's name in any given language. This \\ defines a uniform acronym in all languages.) \\ MPC Multi-Purpose Canister \\ NIST National Institute of Standards and Technology \\ NTSB National Transportation Safety Board \\ OFA Optimized Fuel Assembly \\ PB Personnel Barrier \\ PNNL Pacific Northwest National Laboratory
}


PWR Pressurized Water Reactor

SFPO USNRC Spent Fuel Project Office

SNF Spent nuclear fuel

USNRC United States Nuclear Regulatory Commission 